\title{
A Korean Nationwide Survey for Breakthrough Cancer Pain in an Inpatient Setting
}

\author{
Sun Kyung Baek, MD, PhD ${ }^{1}$ \\ Do Yeun Kim, MD, PhD² \\ Seok Yun Kang, MD, PhD \\ Sun Jin Sym, MD, PhD ${ }^{4}$ \\ Young Sung Kim, MD \\ June Young Lee, $\mathrm{PhD}^{6}$
}

\section{Purpose}

We evaluated the prevalence and characteristics of breakthrough cancer pain (BTcP) in Korean patients admitted with cancer pain.

\section{Materials and Methods}

In-hospital patients with cancer pain completed a questionnaire concerning severity of background cancer pain (BCP), prevalence and treatment for BTcP, sleep disorders, and satisfaction with cancer pain treatment. Medical records showing medications for $\mathrm{BCP}$ and $\mathrm{BTCP}$ were also evaluated.

\section{Results}

Total 609 patients with controlled BCP enrolled. Mean age of the patients was 59.5 years old, and 59\% were male. Of all patients, 177 (29\%) complained of BTcP. No clinical characteristic predicted BTcP. Of the 177 patients with BTcP, 56\% did not receive treatment for BTcP. Patients with BTcP showed significant association with a sleep disorder and dissatisfaction with pain control, compared to those without BTcP $(p<0.0001$ and $p=0.0498$, respectively). Oxycodone-immediate release was the most commonly used short-acting analgesic, followed by intravenous morphine.

\section{Conclusion}

The prevalence of BTcP was $29 \%$ in patients admitted with controlled BCP. Although the patients had well-controlled BCP, BTcP showed association with a lower quality of life in patients with cancer. More medical attention is needed for detection and management of BTcP.

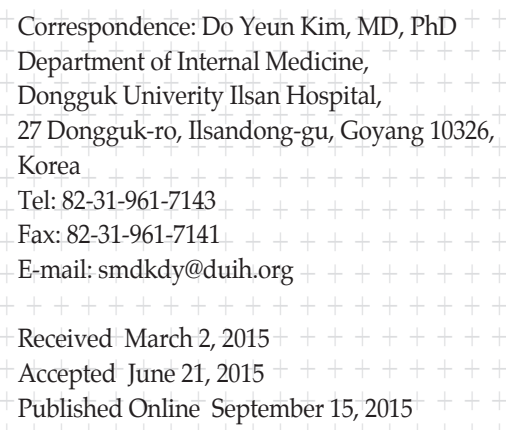

Key words

Breakthrough cancer pain, Prevalence, Characteristics, Quality of life

\section{Introduction}

Pain is a significant problem in cancer patients and is often the most feared aspect of the disease; thus, effective analgesia is an essential component of pain management. Cancer patients require a comprehensive pain treatment plan that not only addresses the moderate to severe background cancer pain (BCP) but also provides adequate management of breakthrough cancer pain $(\mathrm{BTcP})$ that has a separate and characteristically different presentation [1].

$\mathrm{BTcP}$ has different clinical and literature definitions [1]. BTcP was initially defined by Portenoy and Hagen in 1989 [2] as "a transitory flare of pain in the setting of chronic pain 
managed with opioid therapy" [3]. According to more recent and comprehensive definitions, "BTcP is a transient exacerbation of pain that occurs either spontaneously or in relation to a specific predictable or unpredictable trigger, despite relatively stable and adequately controlled BCP" [4]. As there is a lack of consistency in use of the term BTcP, most studies did not report the diagnostic criteria used in screening patients for BTcP or included patients with inadequately controlled $\mathrm{BCP}[3,5]$. Indeed, $\mathrm{BTCP}$ is widely used to describe any exacerbation of pain in patients with $\mathrm{BCP}$ or intermittent episodes of pain in patients without BCP.

$\mathrm{BTcP}$ differs from $\mathrm{BCP}$ due to its high intensity (numerical rating score [NRS], 7), the short time interval between onset and peak intensity (median interval, 3 minutes to peak pain), short duration (median, 30 to 60 minutes), potential recurrence over 24 hours (3-4 daily episodes), and non-responsiveness to treatments for $\mathrm{BCP}[3,6-9]$.

BTcP impacts patient's quality of life (QoL). Because patients with $\mathrm{BTcP}$ are often less satisfied with their analgesic therapy, their functioning decreases due to pain, and they may also experience social and psychosocial consequences, such as increased anxiety and depression [8]. According to a large European observation study and a Canadian study, $\mathrm{BTcP}$ impacts daily living including normal work, mood, sleep, and walking activities [10,11].

Despite the increasing awareness of the need for evaluation and management of $\mathrm{BTCP}$, only a few studies have investigated BTcP in Korea. We conducted this study to characterize the prevalence and treatments for $\mathrm{BTcP}$ in Korea, identify factors associated with the prevalence of $\mathrm{BTcP}$, and to show the impact of BTcP on QoL.

\section{Materials and Methods}

This study was approved by the institutional review board of each participating hospital. This multicenter nationwide study was conducted from 11 September 2010, to 14 October 2010 in 78 hospitals using a patient questionnaire and retrospective review of medical records. The survey instrument was developed by the palliative care committee of the Korean Cancer Study group. Inclusion criteria were hospitalized patients whose $\mathrm{BCP}$ is controlled to a mild degree (NRS 3 or less), were receiving analgesic treatments, with a history of hospitalization for $>4$ days. Patients aged $\geq 20$ years who signed a consent form were eligible for participation. Patients who could not voluntarily participate in the survey due to clouded consciousness or who did not want to participate were ineligible.

Patients completed a questionnaire including (1) average pain intensity for the last 24 hours; (2) prevalence, frequency, and treatments for BTcP; (3) time interval between onset and treatment of breakthrough pain; (4) sleep disorder; and (5) satisfaction with pain treatment for the last week. Sleep disorder was defined as the frequency of unexpected awakening from sleep.

Patients' medical charts were reviewed with respect to pain management until the date the patient entered the study. The following information was obtained from medical records: patient characteristics (age, sex, diagnosis, stage, current treatment, and type of facility), Eastern Cooperative Oncology Group performance status (ECOG PS), and treatments for background pain and BTcP.

The patient's demographic and pain characteristics were summarized by a number of subjects (percentage). Association between the patient characteristics and presence of $\mathrm{BTcP}$ was examined using the chi-square test. A multiple logistic regression model was used to determine factors affecting BTcP. Degrees of difference in patient's QoL (in terms of sleep disorder and satisfaction with pain control) between patients with and without BTcP were examined using crude odds ratios and $95 \%$ confidence intervals. All analyses were performed using SAS ver. 9.4 statistical software (SAS Institute Inc., Cary, NC). A p-value of $<0.05$ was considered significant.

\section{Results}

\section{Patient demographic characteristics}

Of the 1,841 patients with BCP, $496(26.9 \%)$ complained of high NRS pain, and 736 patients (40\%) complained of moderate NRS pain. The remaining 609 patients $(33.1 \%)$ who complained of pain $\leq 3$ NRS were analyzed. Of the 609 patients, 29.1\% complained of BTcP. Percentage of patients $\leq 65$ years was $63 \%$ and that of males was $59 \%$. Most patients $(86 \%)$ were admitted to the general ward, and most were diagnosed with a solid cancer (87\%). Of all patients, $67 \%$ were receiving chemotherapy during the study. Approximately $80 \%$ of patients had stage IV cancer, and $84 \%$ were ECOG PS $<3$ (Table 1).

\section{Predictors for BTcP}

Patients with $\mathrm{BTcP}$ were more frequently admitted to the hospice ward and were less frequently treated with chemotherapy compared to those without BTcP ( $\mathrm{p}=0.0397$ and $\mathrm{p}=0.0642$, respectively) (Table 1 ). However, the results of multivariate analysis showed no significant predictors of 
Table 1. Demographic characteristics of the study subjects

\begin{tabular}{|c|c|c|c|c|}
\hline \multirow{2}{*}{ Variable } & \multicolumn{2}{|c|}{ Breakthrough pain ${ }^{\text {a) }}$} & \multirow{2}{*}{ Total $(n=609)$} & \multirow{2}{*}{ p-value ${ }^{b}$} \\
\hline & Yes $(n=177)$ & No $(n=432)$ & & \\
\hline \multicolumn{5}{|l|}{ Age (yr) } \\
\hline$\leq 65$ & $117(67)$ & $262(61)$ & $379(63)$ & 0.1375 \\
\hline$>65$ & $57(33)$ & $169(39)$ & $226(37)$ & \\
\hline \multicolumn{5}{|l|}{ Sex } \\
\hline Male & $104(59)$ & $253(59)$ & $357(59)$ & 0.9049 \\
\hline Female & $72(41)$ & $179(41)$ & $251(41)$ & \\
\hline \multicolumn{5}{|l|}{ Ward } \\
\hline Hospice & $32(19)$ & $51(12)$ & $83(14)$ & 0.0397 \\
\hline Non-hospice & $138(81)$ & $364(88)$ & $502(86)$ & \\
\hline \multicolumn{5}{|l|}{ Diagnosis } \\
\hline Solid cancerc) & $151(87)$ & $365(87)$ & $516(87)$ & 0.7043 \\
\hline Hematologic malignancy & $21(12)$ & $55(13)$ & $76(13)$ & \\
\hline \multicolumn{5}{|l|}{ Chemotherapy } \\
\hline Yes & $106(61)$ & $287(69)$ & $393(67)$ & 0.0642 \\
\hline No & $67(39)$ & $128(31)$ & $195(33)$ & \\
\hline \multicolumn{5}{|l|}{ Stage } \\
\hline I/II/III & $17(13)$ & $57(17)$ & $74(16)$ & 0.2497 \\
\hline IV & $115(87)$ & $274(83)$ & $389(84)$ & \\
\hline \multicolumn{5}{|l|}{ ECOG PS } \\
\hline $0,1,2$ & $104(76)$ & $276(81)$ & $380(80)$ & 0.2477 \\
\hline 3,4 & $32(24)$ & 64 (19) & $96(20)$ & \\
\hline
\end{tabular}

ECOG PS, Eastern Cooperative Oncology Group performance status. ${ }^{a}$ Values are presented as numbers of subjects $(\%)$, ${ }^{b}$ p-values by chi-square test, ${ }^{c}$ Solid cancer included epithelial originating (lung, stomach, colorectal, breast, esophagus, etc.) and mesenchymal originating cancer.

Table 2. Risk factors for predicting breakthrough cancer pain (BTcP)

\begin{tabular}{lccccc} 
Variable $^{\mathfrak{a})}$ & Coefficient & S.E. & aOR & 95\% CI (aOR) & p-value ${ }^{\mathrm{b})}$ \\
Age $(\leq 65 \mathrm{yr})$ & 0.3429 & 0.2418 & 1.409 & $0.88-2.26$ & 0.1561 \\
Female sex & 0.0313 & 0.2337 & 1.032 & $0.65-1.63$ & 0.8936 \\
Department (non-hospice) & 0.0253 & 0.3602 & 1.026 & $0.51-2.08$ & 0.9439 \\
Diagnosis (solid cancer) & 0.5427 & 0.5745 & 1.721 & $0.56-5.31$ & 0.3448 \\
Current treatment (no) & 0.2967 & 0.2601 & 1.345 & $0.81-2.24$ & 0.2541 \\
Stage (IV) & 0.2259 & 0.3346 & 1.253 & $0.65-2.42$ & 0.4997 \\
ECOG PS (3-4) & 0.3761 & 0.2977 & 1.457 & $0.81-2.61$ & 0.2065 \\
\hline
\end{tabular}

S.E., standard error; aOR, adjusted odds ratio; CI, confidence interval; ECOG PS, Eastern Cooperative Oncology Group performance status. ${ }^{\mathrm{a})}$ Reference: age (> 65 years), sex (male), department (hospice), diagnosis (hematological malignancy), current treatment (yes), stage (I/II/III), ECOG PS $(0,1$, and 2), b) p-values by multiple logistic regression analysis.

BTcP frequency (Table 2).

The frequency of BTcP per day was 73 patients complained once, 62 complained twice, 25 patients complained three times, 11 patients complained four times, and six patients complained more than five times. The mean and median frequencies of $\mathrm{BTCP}$ were 1.95 and 2, respectively. 
Table 3. Associations between breakthrough cancer pain (BTcP) and quality of life

\begin{tabular}{|c|c|c|c|c|c|}
\hline \multirow{2}{*}{ Variable } & \multicolumn{2}{|c|}{ Breakthrough pain ${ }^{\text {a) }}$} & \multirow{2}{*}{ Total $(n=609)$} & \multirow{2}{*}{ p-value ${ }^{b)}$} & \multirow{2}{*}{ cOR $(95 \%$ CI $)$} \\
\hline & Yes $(n=177)$ & No $(n=432)$ & & & \\
\hline \multicolumn{6}{|l|}{ Sleep disorder } \\
\hline 1 or under & $125(71)$ & 393 (91) & $518(85)$ & $<0.0001$ & 1.0 \\
\hline 2 or more & $52(29)$ & $37(9)$ & $89(15)$ & & $4.42(2.77-7.05)$ \\
\hline \multicolumn{6}{|l|}{ Pain control } \\
\hline Dissatisfaction & $23(13)$ & $34(8)$ & $57(9)$ & 0.0498 & $1.74(1.00-3.06)$ \\
\hline Satisfaction & $154(87)$ & 397 (92) & $551(91)$ & & 1.0 \\
\hline
\end{tabular}

cOR, crude odds ratio; CI, confidence interval. ${ }^{a}$ Values are presented as numbers of subjects $(\%)$, b) p-values by chi-square test.

Table 4. Associations between long-acting and short-acting analgesics

\begin{tabular}{|c|c|c|c|c|c|c|}
\hline \multirow{2}{*}{$\begin{array}{l}\text { Long-acting } \\
\text { analgesics }\end{array}$} & \multirow{2}{*}{$\begin{array}{l}\text { Short-acting } \\
\text { analgesics }\end{array}$} & \multicolumn{2}{|c|}{ Breakthrough pain ${ }^{\text {a) }}$} & \multirow{2}{*}{ Total $(n=609)$} & \multirow{2}{*}{ p-value ${ }^{\text {b) }}$} & \multirow{2}{*}{ cOR $(95 \% \mathrm{CI})$} \\
\hline & & Yes $(n=177)$ & No $(n=432)$ & & & \\
\hline \multirow[t]{2}{*}{ Morphine } & Morphine & $3(38)$ & $2(9)$ & $5(17)$ & 0.1020 & $6.00(0.78-46.14)$ \\
\hline & No morphine & $5(63)$ & $20(91)$ & $25(83)$ & & 1.0 \\
\hline \multirow[t]{2}{*}{ Fentanyl patch } & Actiq & 0 & $3(2)$ & $3(1)$ & 0.5528 & - \\
\hline & No actiq & $80(100)$ & $157(98)$ & 237 (99) & & - \\
\hline \multirow[t]{2}{*}{ Hydro-morphone } & Hydromorphone IR & $2(15)$ & $1(4)$ & $3(8)$ & 0.2421 & $4.73(0.39-57.70)$ \\
\hline & No hydromorphone IR & $11(85)$ & $26(96)$ & $37(92)$ & & 1.0 \\
\hline \multirow[t]{2}{*}{ Oxycodone } & Oxycodone IR & $18(31)$ & $20(13)$ & $38(18)$ & 0.0038 & $2.85(1.38-5.90)$ \\
\hline & No oxycodone IR & $41(69)$ & $130(87)$ & $171(82)$ & & 1.0 \\
\hline \multirow[t]{2}{*}{ Tramadol } & Tramadol & 0 & $1(4)$ & $1(3)$ & 1.0000 & - \\
\hline & No tramadol & $6(100)$ & $23(96)$ & $29(97)$ & & - \\
\hline
\end{tabular}

cOR, crude odds ratio; CI, confidence interval; IR, immediate release. ${ }^{a}$ Values are presented as numbers of subjects (\%),

b) $\mathrm{p}$-values by Fisher exact test.

\section{Impact of BTcP on QoL}

Patients with BTcP 4.42 times more frequently complained sleep disorder, which caused patients to wake up two or more times during sleep, compared to those without BTcP $(\mathrm{p}<0.0001)$ (Table 3). And they were 1.74 times more unsatisfied with their pain control than patients who did not have BTcP ( $\mathrm{p}=0.0498)$ (Table 3).

\section{4. $\mathrm{BTcP}$ treatment}

According to the patient questionnaire results, $77 \%$ of patients with $\mathrm{BTcP}$ answered that they were treated with a short-acting analgesic during an attack. A total of 110 patients answered that they were treated with a short-acting analgesic within 10 minutes, 29 patients within 11 to 20 minutes, five patients within 11 to 20 minutes, three patients within 31 to 60 minutes, and six patients after 60 minutes.

According to the medical records, 77 of 177 patients (44\%) with BTcP were prescribed short-acting analgesics on the day of their enrollment in this study. Thirty-six patients were treated with oxycodone-immediate release (IR), 30 patients with morphine (29 intravenous morphine and one S-morphine), six patients with tramadol, three with hydromorphone, one with Actiq, and one with acetaminophen. There was no clinical predictor for the reason why patients did not take and short-acting analgesics. Concordance between longacting and short-acting analgesics was analyzed. A cognate short-acting analgesic, such as oxycodone-IR, was more frequently prescribed for patients treated with oxycodone as a long-acting analgesic $(\mathrm{p}=0.0038)$ (Table 4$)$. 


\section{Discussion}

Recent surveys have reported BTcP prevalence of 28\%-71\% for in-patients with cancer [1]. Here, we reported prevalence of $29 \%$ among hospitalized cancer pain patients. This is a lower percentage than previously reported [12-14], although Hagen et al. [15] reported that $28 \%$ of patients with cancer pain have BTcP. Nationwide surveys conducted in Korea between 2001 and 2006 reported BTcP incidence of 35\% [12]. One explanation for the discrepancy may be different inclusion criteria. Two surveys conducted in 2001 and 2006 enrolled cancer pain patients who visited the outpatient clinic or were admitted in hospital. These also included patients with uncontrolled $\mathrm{BCP}$, and patients with advanced stage cancer and poor PS [12]. In comparison with previous studies, the current survey, conducted in 2010, only enrolled hospitalized patients with controlled $\mathrm{BCP}$, as the exact definition of BTcP.

Actually, prevalence of BTcP varies in different settings. The lowest prevalence rates were detected in studies conducted in out-patient clinics and the highest were reported in studies conducted in the hospice ward [1]. Hospitalized patients who maintained generally good PS and received chemotherapy were enrolled in this study. This setting could explain the lower prevalence of BTcP. Another possible explanation for the lower prevalence of $\mathrm{BTcP}$ in the current study is that patients might have recall bias. As a matter of fact, $77 \%$ of our patients answered that they have received short-acting analgesics for BTcP but the medical records reported that only $44 \%$ of the patients were treated with short-acting analgesics. Thus, patients with BTcP may not have reported their pain because of difference between patients' memory of pain and the medical record of the study day. In addition, as no validated $\mathrm{BTCP}$ assessment tool was available at the time of this study, we could not reliably determine the prevalence of BTcP only by asking patients to describe the frequency, intensity, and duration.

BTcP affects patient's QoL. Bedard et al. [11] reported that BTcP impacts daily living activities of European and Canadian patients and Raj et al. [13] reported that patients with $\mathrm{BTCP}$ have more mood and sleep disturbances and are less able to keep up with normal work. We also found that BTcP was related to dissatisfaction with pain control and sleep disorders. However, there was no available clinical predictor to distinguish patients with and without BTcP. Thus, successful management of $\mathrm{BTCP}$ depends on a combination of adequate assessment and appropriate treatment. A systematic review of the literature in 2010 identified 10 tools for assessment of $\mathrm{BTCP}$, seven of which were discussed but have not been made available and have only been used in one study [14]. Portenoy and Hagen [3] developed the Breakthrough Pain
Questionnaire (BPQ) specifically for assessment of BTcP. This tool assesses the severity, location, pathophysiology, cause, and precipitating and palliative factors for $\mathrm{BTcP}$, as well as its relationship to scheduled analgesic use through patient's self-report. Although the BPQ has been used in epidemiological and pharmaceutical studies, it has not been validated [14]. Hagen et al. [15] developed the Alberta Breakthrough Pain Assessment Tool (ABPAT), using the Delphi process, specifically for assessment of BTcP. This tool was developed for research purposes and was formally validated for clinical use in 2014 [16]. The Episodic Pain Documentation Sheet [17] and a modified version of the original Potenoy and Hagen questionnaire [18] were also developed for assessment of BTCP. However, only the ABPAT has been clinically validated for independent assessment of BTcP.

Oral IR opioid preparations are recommended as first-line therapy for BTcP [19]. Their advantages include easy administration, relatively rapid onset, and extensive experience in use. Morphine, oxycodone, and hydromorphone are available as oral IR preparations in Korea. Most patients were also treated $\mathrm{BTCP}$ with an oral or intravenous opioid. Concordance was observed between oxycodone as a long-acting analgesic and oxycodone IR as a short-acting analgesic because physicians had more experience in prescribing oxycodone as an oral preparation and it could be easily titrated. Many recent studies have suggested that buccal, sublingual, or oral/nasal transmucosal formulations of fentanyl are effective for BTcP $[9,20,21]$. Fentanyl is a highly lipophilic synthetic opioid, which shows rapid diffusion across the blood brain barrier to elicit a rapid pain response [22]. A recent study demonstrated that physicians are more aware of the benefits of fentanyl, as a higher proportion of patients are prescribed this agent for control of BTcP [11]. Fentanyl (Actiq) was only prescribed to $1.2 \%$ of patients in our study. In 2010, Korean physicians had limited options for treatment of BTcP. Actiq was first available for use in Korea in 2008 and Fentora and Abstral became available in 2014. Therefore, nowadays, with an established definition of BTcP, different assessment tools, and many treatment options for $\mathrm{BTcP}$, this result did not coincide exactly with treatment of BTcP. However, this study might be useful in showing the state of epidemiology and clinical significance of BTcP and help support further studies in Korea.

This study had some limitations related to the methods used for classification of patients with BTcP. The study was designed to describe the epidemiology and pain management strategy for patients with cancer in Korea with special emphasis on the prevalence and treatment patterns for cancer pain in 2010. The variables and measurements used were most appropriate for $\mathrm{BCP}$, not BTcP. Therefore, this study did not report subtypes of $\mathrm{BTcP}$ such as spontaneous or incidental type or the impact of $\mathrm{BTcP}$ on daily living. In 
addition, we rely solely on patient self-reporting techniques, which can be inaccurate, as patients are not always specific when reporting their pain, and we did not use a BTcP-specified assessment tool, as no validated assessment tool for BTcP was available at the time of the study. Neverthless, this study presents the first report about BTcP in Korea based on a valid definition of BTcP.

\section{Conclusion}

In summary, approximately $30 \%$ of hospitalized patients with adequately controlled BCP complained of BTcP. Unfortunately, there were no clinical factors predicting the presence of BTcP, and BTcP impacted QoL of cancer patients with controlled BCP. Therefore, physicians should perform more appropriate evaluation, and provide adequate management of BTCP in cancer patients with controlled BCP.

This is the first study to show the prevalence and clinical characteristics of BTcP in Korea based on a valid definition of BTcP. Further advances in BTcP diagnosis and treatment, as well as knowledge of predictors will continue to inform us about the evolving, complex nature of cancer pain classification and management.

\section{Conflicts of Interest}

This research was supported financially by grants from the Johnson \& Johnson family of companies, whose role was restricted and did not involve providing assistance to the investigators in the conception, conduct, and analysis of the study.

\section{Acknowledgments}

We thank all institutions and physicians participating in this survey. Participating institutions are listed as follows.

Ajou University Medical Center, Asan Medical Center, Baptist Hospital, Bobath Memorial Hospital, Bohun Hospital (Daegu), Bohun Hospital (Gwangju), Bohun Hospital (Seoul), Bundang CHA Hospital, Bundang Jesaeng Hospital, Busan Medical Center, Busan St. Mary's Hospital, Cheongju St. Mary's Hospital, Chonbuk National University Hospital, Chonnam National University Hwansun Hospital, Chosun
University Hospital, Chung-Ang University Hospital, Chungbuk National University Hospital, Chungnam National University Hospital, Daegu Fatima Hospital , Daegu Catholic University Medical Center, Dankook University Hospital, Dong-A University Hospital, Dongguk University Ilsan Hospital, Gyeongsang National University Hospital, Eulji University Hospital (Daejeon), Eulji University Hospital (Seoul) Medical Center, Ewha Womens University Mokdong Hospital, Gachon University Gil Hospital, Good Samaritan Hospital, Korea Cancer Center Hospital, Hanil Hospital, Hallym University Medical Center, Hanyang University Hospital, Inha University Hospital, Inje University Haeundae Paik Hospital, Inje University Sanggye Paik Hospital, Inje University Seoul Paik Hospital, Inje University Busan Paik Hospital, Kangbuk Samsung Hospital, Keimyung University Dongsan Medical Center, Konkuk University Medical Center, Konyang University Hospital, Korea University Anam Hospital, Korea University Guro Hospital, Kosin Universiy Gospel Hospital, Kyung Hee University Hospital at Gangdong, Kyungpook National University Hospital, Medi-Yin Hospital, Myongji Hospital, National Health Insurance Servive Ilsan Hospital, Pusan National University Hospital, Pusan National University Yangsan Hospital, SAM Anyang Hospital, Saemyung Hospital, Samgsung Medical Center, Seoul Medical Center, Seoul Metropolitan Seobuk Hospital, Seoul National University Bundang Hospital, Severance Hospital, SMG-SNU Boramae Medical Center, Soonchunhyang University Hospital, Soonchunhyang University Bucheon Hospital, Soonchunhyang University Cheonan Hospital, St. Carollo Hospital, The Catholic University of Korea Bucheon St. Mary's Hospital, The Catholic University of Korea Daejeon St. Mary's Hospital, The Catholic University of Korea Incheon St. Mary's hospital, The Catholic University of Korea Seoul St. Mary's Hospital, The Catholic University of Korea Yeouido St. Mary's Hospital, The Catholic University of Korea St. Paul's Hospital, The Catholic University of Korea Uijeongbu St. Mary's Hospital, Wonju Christian Hospital, Wonkwang University Hospital, Yeungnam University Hospital. 


\section{References}

1. Deandrea S, Corli O, Consonni D, Villani W, Greco MT, Apolone G. Prevalence of breakthrough cancer pain: a systematic review and a pooled analysis of published literature. J Pain Symptom Manage. 2014;47:57-76.

2. Portenoy RK, Hagen NA. Breakthrough pain: definition and management. Oncology (Williston Park). 1989;3(8 Suppl): 25-9.

3. Portenoy RK, Hagen NA. Breakthrough pain: definition, prevalence and characteristics. Pain. 1990;41:273-81.

4. Davies AN, Dickman A, Reid C, Stevens AM, Zeppetella G; Science Committee of the Association for Palliative Medicine of Great Britain and Ireland. The management of cancer-related breakthrough pain: recommendations of a task group of the Science Committee of the Association for Palliative Medicine of Great Britain and Ireland. Eur J Pain. 2009;13:331-8.

5. Zeppetella G, O'Doherty CA, Collins S. Prevalence and characteristics of breakthrough pain in cancer patients admitted to a hospice. J Pain Symptom Manage. 2000;20:87-92.

6. Davies A, Zeppetella G, Andersen S, Damkier A, Vejlgaard T, Nauck F, et al. Multi-centre European study of breakthrough cancer pain: pain characteristics and patient perceptions of current and potential management strategies. Eur J Pain. 2011; 15:756-63.

7. Portenoy RK, Bruns D, Shoemaker B, Shoemaker SA. Breakthrough pain in community-dwelling patients with cancer pain and noncancer pain, part 2: impact on function, mood, and quality of life. J Opioid Manag. 2010;6:109-16.

8. Portenoy RK, Payne D, Jacobsen P. Breakthrough pain: characteristics and impact in patients with cancer pain. Pain. 1999; 81:129-34.

9. Zucco F, Bonezzi C, Fornasari D. Breakthrough cancer pain (BTcP): a synthesis of taxonomy, pathogenesis, therapy, and good clinical practice in adult patients in Italy. Adv Ther. 2014; 31:657-82.

10. Davies A, Buchanan A, Zeppetella G, Porta-Sales J, Likar R, Weismayr $W$, et al. Breakthrough cancer pain: an observational study of 1000 European oncology patients. J Pain Symptom Manage. 2013;46:619-28.

11. Bedard G, Davies A, McDonald R, Hawley P, Buchanan A, Popovic $\mathrm{M}$, et al. Breakthrough cancer pain: a comparison of surveys with European and Canadian patients. Support Care Cancer. 2015;23:791-6.

12. Hong SH, Roh SY, Kim SY, Shin SW, Kim CS, Choi JH, et al. Change in cancer pain management in Korea between 2001 and 2006: results of two nationwide surveys. J Pain Symptom Manage. 2011;41:93-103.

13. Raj SX, Thronaes M, Brunelli C, Hjermstad MJ, Klepstad P, Kaasa S. A cross-sectional study on prevalence of pain and breakthrough pain among an unselected group of outpatients in a tertiary cancer clinic. Support Care Cancer. 2014; 22:1965-71.

14. Haugen DF, Hjermstad MJ, Hagen N, Caraceni A, Kaasa S; European Palliative Care Research Collaborative (EPCRC). Assessment and classification of cancer breakthrough pain: a systematic literature review. Pain. 2010;149:476-82.

15. Hagen NA, Stiles C, Nekolaichuk C, Biondo P, Carlson LE, Fisher $\mathrm{K}$, et al. The Alberta Breakthrough Pain Assessment Tool for cancer patients: a validation study using a delphi process and patient think-aloud interviews. J Pain Symptom Manage. 2008;35:136-52.

16. Sperlinga R, Campagna S, Berruti A, Laciura P, Ginosa I, Paoletti $S$, et al. Alberta Breakthrough Pain Assessment Tool: a validation multicentre study in cancer patients with breakthrough pain. Eur J Pain. 2015;19:881-8.

17. Zeppetella G, Ribeiro MD. Episodic pain in patients with advanced cancer. Am J Hosp Palliat Care. 2002;19:267-76.

18. Caraceni A, Bertetto O, Labianca R, Maltoni M, Mercadante S, Varrassi G, et al. Episodic (breakthrough) pain prevalence in a population of cancer pain patients. Comparison of clinical diagnoses with the QUDEI: Italian questionnaire for intense episodic pain. J Pain Symptom Manage. 2012;43:833-41.

19. Schug SA, Chandrasena C. Pain management of the cancer patient. Expert Opin Pharmacother. 2015;16:5-15.

20. Zeppetella G, Davies AN. Opioids for the management of breakthrough pain in cancer patients. Cochrane Database Syst Rev. 2013;10:CD004311.

21. Mercadante S. Pharmacotherapy for breakthrough cancer pain. Drugs. 2012;72:181-90.

22. Davis MP. Fentanyl for breakthrough pain: a systematic review. Expert Rev Neurother. 2011;11:1197-216. 